\title{
Long gamma-ray bursts as tracers of star formation
}

\author{
Jure Japelj* \\ Anton Pannekoek Institute for Astronomy, University of Amsterdam, Science Park 904, 1098 XH \\ Amsterdam, The Netherlands \\ E-mail: j.japelj@uva.nl
}

\begin{abstract}
An important part of studying long gamma-ray bursts (GRBs) lies in studying their environments. On the one hand, GRB host galaxies provide us with clues to better understand these extreme explosions. On the other hand, we can use GRBs to pinpoint faint star-forming galaxies and to trace star-formation across the Universe. In the recent years a number of unbiased GRB samples have been compiled. Thanks to these samples, which are highly complete in redshift, we can finally understand the intrinsic properties of the GRB host galaxy population. In this review we give a brief summary of the main characteristics of GRB host galaxies and their relation to the field star-forming population. We mainly focus on the results we obtained by studying the complete BAT6 sample.
\end{abstract}

XII Multifrequency Behaviour of High Energy Cosmic Sources Workshop 12-17 June, 2017

Palermo, Italy

${ }^{*}$ Speaker. 


\section{Introduction}

Long gamma-ray bursts (GRBs) are short and luminous cosmological events which are observed up to $z \lesssim 9$ [1]. A short burst in gamma rays is usually followed by a broadband afterglow emission, which, depending on the wavelength, can be observed for days to weeks after the initial explosion (see review in [2]). The association of GRBs with supernova emission [3] established their connection to the deaths of young, massive stars. A detection of the afterglow emission enables to pinpoint the location of a GRB in the sky and allows subsequent observations of galaxies in which GRBs occur.

Studying GRBs is closely connected to studying their environments [4]. Being linked to massive progenitor stars, GRBs originate in star-forming regions in galaxies at cosmological distances. The afterglow light that we observe is imprinted by properties of gas and dust in the line-of-sight between us and the point of explosion, including the material in the host galaxy itself. GRBs therefore provide us a unique view into the star-forming regions in the young Universe.

On the other hand, studying the integrated properties of GRB host galaxies is of particular interest for at least two reasons. Firstly, the properties of their host environments can provide us with important clues about the nature of their progenitor stars which are still poorly known. Secondly, because of their link to the young, massive stars, they are tracing current star formation in their host galaxies. As such, they have a potential to be powerful tracers of star formation in the Universe [5], especially since the standard luminosity-selected galaxy surveys have to overcome a number of limitations (e.g. magnitude-limited samples, dust extinction, redshift completeness). The focus of the studies in the recent decade has been to understand the connection between the GRB phenomena and star formation processes and to establish whether GRB host population really represents the star-forming population in the Universe. Especially in recent years a consensus has been reached on the general properties of the population thanks to the compilation of samples highly complete in redshift.

\section{The lessons learned from complete samples}

Early studies of GRB host environments have reached different, often opposite conclusions due to selection of biased samples. For example, samples were usually based on the detected optical afterglow, and were therefore biased against dust-obscured systems, also known as dark GRBs [6]. Thanks to the Swift mission [7], the number of detected GRBs with a decent localization $(<2 ")$ based on the X-ray afterglow dramatically increased and the detection of an optical afterglow was no longer a priority to identify the host galaxy. In the recent years, several so-called complete samples have been compiled: BAT6 [8], TOUGH [9] and SHOALS [10]. All of these complete samples are composed of Swift GRBs selected according to the preferable observing conditions in such a way that they ensure high redshift completeness yet do not affect the intrinsic properties of the GRB population.

To understand whether GRBs trace star formation, their host galaxies have to be characterized and compared to the properties of field star-forming galaxies. In particular we are interested in measuring luminosities, stellar masses $\left(M_{\star}\right)$, star-formation rates (SFR) and gas-phase metallicities. Proper measurements require a well sampled photometric SEDs (extending into the infrared) and 

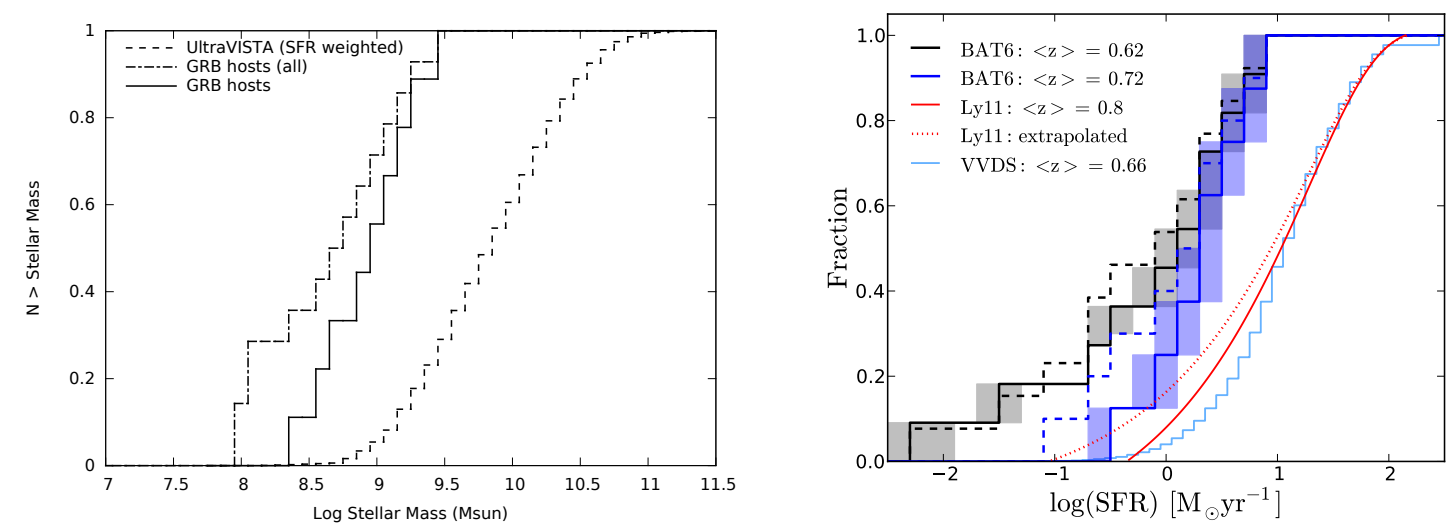

Figure 1: Comparison of stellar masses (left; [12]) and SFRs (right; [13]) of the $z<1$ GRB hosts of the complete BAT6 sample and field star-forming galaxies. GRBs are preferentially found in low-mass, low-SFR galaxies.

high-quality spectra. The VLT/X-shooter instrument [11], with its large wavelength range (300 $2500 \mathrm{~nm}$ ) and high efficiency, has proved to be the ideal instrument for observations of GRB host galaxies. Complete samples with fully characterized galaxies have been obtained for the $z \lesssim 2$ redshift range. At higher redshifts spectroscopic observations become very time-demanding due to intrinsic faintness of the hosts and consequently most studies focus on photometric properties.

Special attention has to be given to the comparison field-galaxy samples. As GRB host galaxies are intrinsically faint and of low mass, it is hard to find comparison samples of similar completeness even at low redshifts $[12,13]$. A proper way of dealing with this is to cut the GRB host samples below the completeness limits of the field survey, resulting in low-number statistics. Finally, the comparison is typically done in the following way. We want to test the hypothesis that GRBs trace SFR. Therefore the probability of finding a GRB in a galaxy is, in the first order, proportional to the SFR. The comparison of a certain property, e.g. stellar mass, is therefore done against SFR-weighted field samples. If the stellar mass distribution for GRB hosts and SFRweighted distribution of stellar mass of the field galaxies is different, then GRBs are not unbiased tracers of SFR.

\subsection{Stellar masses, SFRs and metallicities}

A thorough study of the $z<1$ host galaxies of the BAT6 complete sample revealed that GRBs preferentially select faint, low-mass and low-SFR galaxies [12,13] and are therefore not unbiased tracers of star formation at low redshifts (see Figure 1). An increased incidence of high specific SFR (sSFR; SFR divided by stellar mass) galaxies, or starbursts, was found among hosts with respect to the field galaxies [13], though this result should be confirmed on a larger sample study.

A special attention has been given to understand the metallicity distribution of the hosts (Figure 2), given its importance in stellar evolution (see 2.2). Studies have used both direct and indirect methods to show that GRBs prefer low-metallicity environment. Firstly, since stellar mass and metallicity are positively correlated in the so-called mass-metallicity relation [14], the dearth of high-mass hosts at $z<1$ points to low metallicities. Directly measured metallicities from nebular 

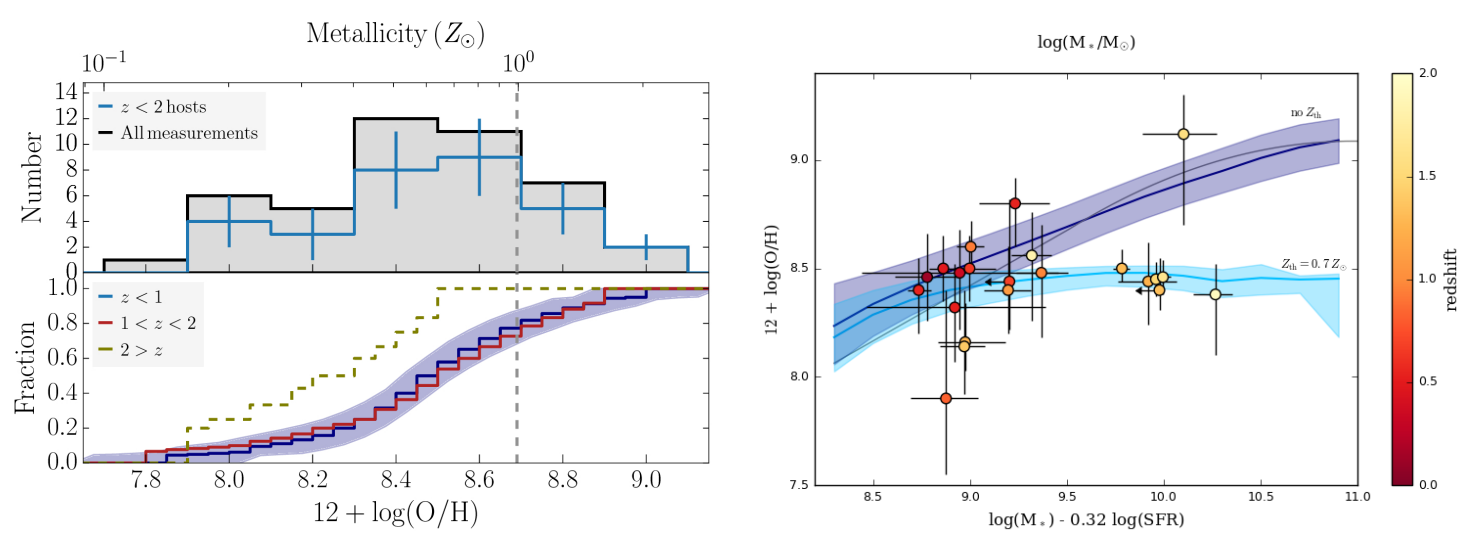

Figure 2: Left: gas-phase metallicity distribution of GRB hosts [15]. Dashed vertical line indicates solar metallicity. Right: GRBs from BAT6 complete sample in $z<2$ range are plotted in the fundamental massmetallicity plane [17]. The projection of the plane, including scatter, is plotted in grey region. GRB hosts show a clear offset from the relation and preference for low metallicity environments (cyan).

emission lines showed that the majority of hosts have sub-solar metallicities, though not exclusively $(\approx 15 \%$ of GRB hosts have super-solar metallicities [15]). Metallicity distributions of GRB hosts and field galaxies show a good match until a cutoff value after which the paucity of high-metallicity hosts becomes apparent [13]. Interestingly the low-metallicity preference also reveals itself by plotting GRB hosts in the fundamental mass metallicity plane [16], that is a plane in the stellar mass, SFR and metallicity parameter space which has been shown to hold for star-forming galaxies up to $z \sim 2.5$. While $z<1$ GRB hosts fall onto the plane [13], they show a strong deviation when including $1<z<2$ GRB hosts [17]. Despite all the studies the exact value of metallicity cutoff remains poorly constrained, partly due to different metallicity calibrators used in the literature, and partly due to small samples.

At $z \gtrsim 2$ the spectroscopic observations become sparse and complete samples are investigated via photometric means, i.e. investigating stellar mass and luminosity. The general trend is that with higher redshifts GRB hosts become more and more representative of the field population. This has been shown both by studying the rest-frame near-infrared luminosities of the SHOALS sample [18] and the rest-frame UV luminosities of the TOUGH sample [19]. At $z \gtrsim 5$ the studies are affected by small number statistics and the question whether GRB hosts represent the star-forming population or not is still under debate [19].

\subsection{Implications for progenitors}

By studying GRB host galaxies we gain indirect clues about their progenitor stars. If the preference for high SSFR is confirmed, we would get an evidence that GRB progenitor stars are very young (and therefore massive), because very high values of SSFR are hard to maintain for a long time. The evidence for young stellar progenitors is also given by the observation that GRBs are concentrated on the brightest parts of their host galaxies [20]. The sites with the most recent star formation are expected to be brighter than the sights where some time has already passed from the onset of star-formation (but see [4]). 
The preference for low metallicities has been given a lot of attention due to the strong impact the metallicity has in the process of stellar evolution. Single-star evolution models leading to a GRB explosion indeed predict that such stars should be metal-poor [21, 22]. How metal-poor is however hard to predict, as the exact value of the metallicity that is necessary for a GRB production depends on the detailed treatment of rotation [23]. The restriction on the stellar metallicity is less severe in the case of binary-star evolution models [24]. While host galaxy metallicities are indeed found to be low in general, we cannot ignore the fact that $\sim 15 \%$ of GRBs are found in metal-rich environments. In order to use the metallicity to learn more and possibly discriminate between different models, it will be important to constrain the cutoff metallicity and the shape of the metallicity distribution at the high-metallicity end. Furthermore, it is still not clear whether metallicity really is the only factor affecting the efficiency of GRB formation [4]. Finally, we note that due to the faintness of GRB host galaxies we can only measure gas-phase oxygen abundances which are then used as a proxy for metallicity. In stellar evolution models the metallicity is instead traced (mostly) by iron. The relation between the gas-phase oxygen abundances and the stellar iron-based metallicity is not trivial and the comparison between the measured metallicities and theoretical models is therefore not straightforward.

Even more can be learned about the progenitors by comparing environments of GRBs to those of other extreme transients. Lately, a class of very luminous (superluminous) supernovae (SLSN) has drawn a lot of attention [25]. In particular, hydrogen-poor class of SLSN was preferentially found in low-mass, low-SFR and low-metallicity environments [26, 27]. The comparison to GRB hosts has so far been limited to the $z<1$ range and showed that SLSN hosts are of even lower stellar mass and lower luminosity [27], while they may not be dramatically different in other properties [28]. It will be interesting to see how and if the relation between the host galaxies of GRBs and SLSNe changes when moving towards higher redshifts.

\subsection{High-redshift applications}

The observation that GRB host population is becoming more and more representative of the star-forming galaxies as we move toward higher redshifts is encouraging. GRBs can therefore be used as an independent way to measure cosmic star formation rate at high redshifts [29]. Furthermore, GRBs pinpoint the galaxies which are at the faint end of luminosity function at $z \gtrsim 6$ $[30,31,32]$. Such faint galaxies are usually missed in standard galaxy surveys. If GRB hosts indeed represent the bulk of star-forming population at high redshifts, then by understanding their properties at low redshifts (where we can actually study them in detail), we get to learn about the star-forming population in the (very) young Universe.

\section{Conclusions}

Host galaxies of long gamma-ray bursts are interesting and important astrophysical objects. They provide us with clues about the nature of GRB progenitors. The results of the complete, unbiased samples show them as possible representatives of the bulk of star-forming population at high redshifts. Studying GRB host galaxies may therefore prove invaluable for the understanding of the population of faint galaxies that is believed to be providing the majority of the ionizing photons in the epoch of reionization (e.g. [33]). With the advent of the space-based GRB observatory 
SVOM [34], designed in such a way to detect a larger fraction of high-redshift GRBs, the research of GRBs and their host galaxies will play an important role in our search to understand some of the fundamental questions in modern astrophysics.

\section{Acknowledgments}

The author acknowledges support from NOVA and NWO-FAPESP grant for advanced instrumentation in astronomy.

\section{References}

[1] R. Salvaterra, High redshift Gamma-Ray Bursts, Journal of High Energy Astrophysics 7 (Sept., 2015) 35-43, [1503.03072].

[2] P. Kumar and B. Zhang, The physics of gamma-ray bursts \& relativistic jets, 561 (Feb., 2015) 1-109, [1410.0679].

[3] Z. Cano, S.-Q. Wang, Z.-G. Dai and X.-F. Wu, The Observer's Guide to the Gamma-Ray Burst Supernova Connection, Advances in Astronomy 2017 (2017) 8929054, [1604.03549].

[4] D. A. Perley, Y. Niino, N. R. Tanvir, S. D. Vergani and J. P. U. Fynbo, Long-Duration Gamma-Ray Burst Host Galaxies in Emission and Absorption, 202 (Dec., 2016) 111-142, [1602.00770].

[5] B. E. Robertson and R. S. Ellis, Connecting the Gamma Ray Burst Rate and the Cosmic Star Formation History: Implications for Reionization and Galaxy Evolution, 744 (Jan., 2012) 95, [1109.0990].

[6] T. Krühler, J. Greiner, P. Schady, S. Savaglio, P. M. J. Afonso, C. Clemens et al., The SEDs and host galaxies of the dustiest GRB afterglows, 534 (Oct., 2011) A108, [1108.0674].

[7] N. Gehrels, G. Chincarini, P. Giommi, K. O. Mason, J. A. Nousek, A. A. Wells et al., The Swift Gamma-Ray Burst Mission, 611 (Aug., 2004) 1005-1020.

[8] R. Salvaterra, S. Campana, S. D. Vergani, S. Covino, P. D’Avanzo, D. Fugazza et al., A Complete Sample of Bright Swift Long Gamma-Ray Bursts. I. Sample Presentation, Luminosity Function and Evolution, 749 (Apr., 2012) 68, [1112.1700].

[9] J. Hjorth, D. Malesani, P. Jakobsson, A. O. Jaunsen, J. P. U. Fynbo, J. Gorosabel et al., The Optically Unbiased Gamma-Ray Burst Host (TOUGH) Survey. I. Survey Design and Catalogs, 756 (Sept., 2012) 187, [1205.3162].

[10] D. A. Perley, T. Krühler, S. Schulze, A. de Ugarte Postigo, J. Hjorth, E. Berger et al., The Swift Gamma-Ray Burst Host Galaxy Legacy Survey. I. Sample Selection and Redshift Distribution, 817 (Jan., 2016) 7, [1504.02482].

[11] J. Vernet, H. Dekker, S. D’Odorico, L. Kaper, P. Kjaergaard, F. Hammer et al., X-shooter, the new wide band intermediate resolution spectrograph at the ESO Very Large Telescope, 536 (Dec., 2011) A105, [1110.1944].

[12] S. D. Vergani, R. Salvaterra, J. Japelj, E. Le Floc'h, P. D'Avanzo, A. Fernandez-Soto et al., Are long gamma-ray bursts biased tracers of star formation? Clues from the host galaxies of the Swift/BAT6 complete sample of LGRBs. I. Stellar mass at $z \lesssim 1,581$ (Sept., 2015) A102, [1409.7064]. 
[13] J. Japelj, S. D. Vergani, R. Salvaterra, P. D’Avanzo, F. Mannucci, A. Fernandez-Soto et al., Are long gamma-ray bursts biased tracers of star formation? Clues from the host galaxies of the Swift/BAT6 complete sample of bright LGRBs. II. Star formation rates and metallicities at $z \lesssim 1,590$ (May, 2016) A129, [1604.01034].

[14] C. A. Tremonti, T. M. Heckman, G. Kauffmann, J. Brinchmann, S. Charlot, S. D. M. White et al., The Origin of the Mass-Metallicity Relation: Insights from 53,000 Star-forming Galaxies in the Sloan Digital Sky Survey, 613 (Oct., 2004) 898-913, [astro-ph/ 0405537 ].

[15] T. Krühler, D. Malesani, J. P. U. Fynbo, O. E. Hartoog, J. Hjorth, P. Jakobsson et al., GRB hosts through cosmic time. VLT/X-Shooter emission-line spectroscopy of $96 \gamma$-ray-burst-selected galaxies at 0.1 z 3.6, 581 (Sept., 2015) A125, [1505.06743].

[16] F. Mannucci, R. Salvaterra and M. A. Campisi, The metallicity of the long GRB hosts and the fundamental metallicity relation of low-mass galaxies, 414 (June, 2011) 1263-1268, [1011.4506].

[17] S. D. Vergani, J. Palmerio, R. Salvaterra, J. Japelj, F. Mannucci, D. A. Perley et al., The chemical enrichment of long gamma-ray bursts nurseries up to $z=2,599$ (Mar., 2017) A120, [1701.02312].

[18] D. A. Perley, N. R. Tanvir, J. Hjorth, T. Laskar, E. Berger, R. Chary et al., The Swift GRB Host Galaxy Legacy Survey. II. Rest-frame Near-IR Luminosity Distribution and Evidence for a Near-solar Metallicity Threshold, 817 (Jan., 2016) 8, [1504.02479].

[19] S. Schulze, R. Chapman, J. Hjorth, A. J. Levan, P. Jakobsson, G. Björnsson et al., The Optically Unbiased GRB Host (TOUGH) Survey. VII. The Host Galaxy Luminosity Function: Probing the Relationship between GRBs and Star Formation to Redshift 6, 808 (July, 2015) 73, [1503.04246].

[20] A. S. Fruchter, A. J. Levan, L. Strolger, P. M. Vreeswijk, S. E. Thorsett, D. Bersier et al., Long $\gamma$-ray bursts and core-collapse supernovae have different environments, 441 (May, 2006) 463-468, [astro-ph/0603537].

[21] S.-C. Yoon and N. Langer, Evolution of rapidly rotating metal-poor massive stars towards gamma-ray bursts, 443 (Nov., 2005) 643-648, [astro-ph/0 508242].

[22] S. E. Woosley and A. Heger, The Progenitor Stars of Gamma-Ray Bursts, 637 (Feb., 2006) 914-921, [astro-ph/0508175].

[23] C. Georgy, S. Ekström, G. Meynet, P. Massey, E. M. Levesque, R. Hirschi et al., Grids of stellar models with rotation. II. WR populations and supernovae/GRB progenitors at $Z=0.014,542$ (June, 2012) A29, [1203.5243].

[24] C. L. Fryer, P. A. Mazzali, J. Prochaska, E. Cappellaro, A. Panaitescu, E. Berger et al., Constraints on Type Ib/c Supernovae and Gamma-Ray Burst Progenitors, 119 (Nov., 2007) 1211-1232, [astro-ph/0702338].

[25] A. Gal-Yam, Luminous Supernovae, Science 337 (Aug., 2012) 927, [1208 . 3217 ].

[26] G. Leloudas, S. Schulze, T. Krühler, J. Gorosabel, L. Christensen, A. Mehner et al., Spectroscopy of superluminous supernova host galaxies. A preference of hydrogen-poor events for extreme emission line galaxies, 449 (May, 2015) 917-932, [1409.8331].

[27] S. Schulze, T. Krühler, G. Leloudas, J. Gorosabel, A. Mehner, J. Buchner et al., Cosmic evolution and metal aversion in superluminous supernova host galaxies, 473 (Jan., 2018) 1258-1285, [1612.05978]. 
[28] J. Japelj, S. D. Vergani, R. Salvaterra, L. K. Hunt and F. Mannucci, Taking stock of superluminous supernovae and long gamma-ray burst host galaxy comparison using a complete sample of LGRBs, 593 (Oct., 2016) A115, [1607.01045].

[29] M. D. Kistler, H. Yuksel and A. M. Hopkins, The Cosmic Star Formation Rate from the Faintest Galaxies in the Unobservable Universe, ArXiv e-prints (May, 2013), [1305.1630].

[30] N. R. Tanvir, A. J. Levan, A. S. Fruchter, J. P. U. Fynbo, J. Hjorth, K. Wiersema et al., Star Formation in the Early Universe: Beyond the Tip of the Iceberg, 754 (July, 2012) 46, [1201.6074].

[31] R. Salvaterra, U. Maio, B. Ciardi and M. A. Campisi, Simulating high-z gamma-ray burst host galaxies, 429 (Mar., 2013) 2718-2726, [1212 .0856].

[32] J. T. W. McGuire, N. R. Tanvir, A. J. Levan, M. Trenti, E. R. Stanway, J. M. Shull et al., Detection of Three Gamma-ray Burst Host Galaxies at z 6, 825 (July, 2016) 135, [1512 .07808].

[33] J. Japelj, E. Vanzella, F. Fontanot, S. Cristiani, G. B. Caminha, P. Tozzi et al., Constraints on the Lyman continuum escape fraction for faint star-forming galaxies, 468 (June, 2017) 389-403, [1612.06401].

[34] J. Wei, B. Cordier, S. Antier, P. Antilogus, J.-L. Atteia, A. Bajat et al., The Deep and Transient Universe in the SVOM Era: New Challenges and Opportunities - Scientific prospects of the SVOM mission, ArXiv e-prints (Oct., 2016) , [1610.06892]. 